\title{
AN EIGENFUNCTION EXPANSION FOR A NONSELFADJOINT, INTERIOR POINT BOUNDARY VALUE PROBLEM
}

\author{
BY
}

\author{
ALLAN M. KRALL
}

ABSTRACT. Under discussion is the vector system $L y=y^{\prime}+P y, \sum_{j=0}^{\infty} A_{j}\left(t_{j}\right)$ $=0$, where $\sum_{j=0}^{\infty}\|A\|<\infty$. The eigenvalues for the system are known to be countable and approach $\infty$ in the complex plane in a series of well-defined vertical steps. For each eigenvalue there exists an eigenmanifold, generated by the residue of the Green's function. Further, since the Green's function vanishes near $\infty$ in the complex plane when the path toward $\infty$ is horizontal, the Green's function can be expressed as a series of its residues. This in turn leads to two eigenfunction expansions, one for elements in the domain of the original system, another for elements in the domain of the adjoint system.

1. Introduction. Birkhoff and Langer [1] have long ago discussed various problems associated with the system

$$
L y=y^{\prime}+P y, \quad A_{0} y(0)+A_{1} y(1)=0,
$$

deriving the adjoint system, spectrum, Green's function and also an eigenfunction expansion under certain regularity conditions.

Their work was extended by Cole [3] who introduced interior boundary points, so that the system now had the form

$$
L y=y^{\prime}+P y, \quad \sum_{j=0}^{m} A_{j} y\left(t_{j}\right)=0,
$$

where $t_{0}=0, t_{1}=1$, and $0<t_{j}<1, j=2, \cdots, m$. He also derived the adjoint system, spectrum, Green's function, and an eigenfunction expansion.

Recently, Green and Krall [4] began a systematic study of the system

$$
L y=y^{\prime}+P y, \quad \sum_{j=0}^{\infty} A_{j} y\left(t_{j}\right)=0,
$$

where $t_{0}=0, t_{1}=1$, and $0<t_{j}<1, j=2, \cdots, \infty$, and

$$
\sum_{j=0}^{\infty}\left\|A_{j}\right\|<\infty \text {. }
$$

Received by the editors March 9, 1971.

AMS 1969 subject classifications. Primary 3420, 3430; Secondary 3436.

Key words and phrases. Operator, differential operator, boundary value problem, differential system, nonselfadjoint, Sturm-Liouville problem, adjoint, eigenvalue, spectrum, spectral resolution. 
The boundary points $t_{j}, j=1, \cdots, \infty$, may possibly be dense in the interval $[0,1]$. The adjoint system, spectrum and Green's function were derived, and, in addition, in [7] the author has achieved an eigenfunction expansion in a selfadjoint situation.

The present article derives an eigenfunction expansion in a nonselfadjoint situation.

2. Previous results. We let $H$ denote the Hilbert space of $n$-dimensional, vector valued functions, defined on the interval $[0,1]$ and generated by the inner product

$$
(y, z)=\int_{0}^{1} z^{*}(t) y(t) d t=\sum_{i=1}^{n} \int_{0}^{1} \bar{z}_{i}(t) y_{i}(t) d t .
$$

We let $P$ be a continuous $n \times n$ matrix valued function, and $A_{j}, j=0, \cdots$, $\infty$, be constant $n \times n$ matrices which satisfy

$$
\sum_{j=0}^{\infty}\left\|A_{j}\right\|<\infty
$$

where $\|\cdot\|$ denotes an appropriate matrix norm.

Then $D_{0}$ denotes the collection of all elements $y$ in $H$ which satisfy

(a) $y$ is absolutely continuous on $[0,1]$.

(b) $l y=y^{\prime}+P y$ exists a.e. and is in $H$.

Further, $D$ denotes the collection of all elements $y$ in $H$ which satisfy

(a) $y$ is in $D_{0}$.

(b) $U(y)=\sum_{j=0}^{\infty} A_{j} y\left(t_{j}\right)=0$, where the points $t_{j}, j=0, \cdots, \infty$, are in $[0,1]$, $t_{0}=0$, and $t_{1}=1$.

Finally we define the operator $L$ by letting $L y=l y$ for all $y$ in $D$.

It is easy to show that if $\phi(t)$ is a fundamental matrix for $y^{\prime}+P y=0$, then a fundamental matrix for $y^{\prime}+P y=\lambda y$ is given by

$$
\phi(t, \lambda)=\phi(t) e^{\lambda t} \text {. }
$$

It was shown in [4], that when $A_{0}$ and $A_{1}$ are nonsingular, the spectrum of the closed operator $L$ consists only of eigenvalues, which are bounded in the complex $(\lambda)$ plane to the right and to the left, which approach $\pm \infty$ in a vertical strip in a manner which leaves them evenly spaced, and with no finite limit points. The eigenvalues $\lambda$ satisfy the equation

$$
\operatorname{det}\left[\sum_{j=0}^{\infty} A_{j} \phi\left(t_{j}\right) e^{\lambda t_{j}}\right]=0 .
$$

For each $\lambda$, not an eigenvalue, $(L-\lambda I)^{-1}$ exists and is generated by a Green's function, given by 


$$
G(\lambda, t, s)=\phi(t) e^{\lambda t} U\left(\phi(t) e^{\lambda t}\right)^{-1} M(t, s) \phi(s)^{-1} e^{-\lambda s},
$$

where

$$
\begin{aligned}
M(t, s) & =\sum_{t_{k} \leq s} A_{k} \phi\left(t_{k}\right) e^{\lambda t_{k}}, \quad s<t, \\
& =-\sum_{t_{k}>s} A_{k} \phi\left(t_{k}\right) e^{\lambda_{t}}, \quad s>t .
\end{aligned}
$$

The Green's function has the properties:

1. $G(\lambda, t, s)$ is defined for all $t, s$ in $[0,1] \times[0,1], t \neq s$.

2. $G(\lambda, t+, t)-G(\lambda, t-, t)=I$.

3. $\partial G(\lambda, t, s) / \partial t+P(t) G(\lambda, t, s)=\lambda G(\lambda, t, s)$, a.e.

4. $\sum_{j=0}^{\infty} A_{j} G\left(\lambda, t_{j}, s\right)=0, s \neq t_{j}, j=0, \cdots, \infty$.

5. $-\partial G(\lambda, t, s) / \partial s+G(\lambda, t, s) P(s)=\lambda G(\lambda, t, s)$, a.e.

6. $G\left(\lambda, t, t_{j}+\right)-G\left(\lambda, t, t_{j}-\right)=\phi(t) e^{\lambda t} U\left(\phi(t) e^{\lambda t}\right)^{-1} A_{j}, j=0, \cdots, \infty$.

The operator $(L-\lambda I)^{-1}$, given by

$$
(L-\lambda I)^{-1} f(t)=\int_{0}^{1} G(\lambda, t, s) f(s) d s,
$$

is compact.

Finally, the adjoint operator $L^{*}$ exists. Its domain $D^{*}$ consists of all elements $z$ in $H$ which satisfy

(a) For some parametric vector $\phi$,

$$
z-\sum_{j=0}^{\infty} A_{j}^{*} \phi \chi_{\left(t_{j, 1}\right]}
$$

is absolutely continuous on $[0,1] .\left(\chi_{A}\right.$ is the characteristic function of the set A.)

(b) $-z^{\prime}+P^{*}$ exists a.e. and is in $H$.

(c) $z\left(0_{-}\right)=0, z(1+)=0$.

Condition (a) implies that $z$ has a jump discontinuity at each boundary point $t_{j}$

$$
z\left(t_{j}+\right)-z\left(t_{j}-\right)=A_{j}^{*} \phi
$$

Then for all $z$ in $D^{*}$,

$$
L^{*} z=-z^{\prime}+P^{*} z, \text { a.e. }
$$

3. Eigenmanifolds. The eigenvalues of $L$ are the roots of the equation

$$
\operatorname{det}\left[\sum_{j=0}^{\infty} A_{j} \phi\left(t_{j}\right) e^{\lambda t_{j}}\right]=0 .
$$


But, unlike selfadjoint problems, if $\lambda$ is an $m$-fold zero of the equation above, the $m$-dimensional eigenmanifold associated with it may be in the form of an eigenfunction chain. This is also true for the adjoint operator.

Since the adjoint operator is not a differential operator in the usual sense, before we look at these chains, we shall consider solutions to the adjoint equations $\left[L^{*}-\bar{\lambda} I\right] v=0$, and $\left[L^{*}-\bar{\lambda} I\right] v=f$.

3.1. Theorem. The solution to the problem

(a) $-z^{\prime}+P^{*} z=\bar{\lambda} z$, a.e.,

(b) $z\left(t_{j}+\right)-z\left(t_{j}-\right)=A_{j}^{*} \phi, j=0, \cdots, \infty$, for some parameter $\phi$,

(c) $z(0-)=0$,

is given by

$$
z(t)=\phi^{*}(t)^{-1} e^{-\bar{\lambda} t} \int_{0-}^{t} \phi^{*}(s) e^{\bar{\lambda} s} d F(s) \phi,
$$

where $F$ is a function of bounded variation satisfying $F\left(0_{-}\right)=0$,

$$
d F(t)=\left\{\begin{array}{ll}
0, & t \neq t_{j}, \\
A_{j}^{*}, & t=t_{j},
\end{array} \quad j=0, \cdots, \infty .\right.
$$

Proof. This is obvious by inspection.

3.2. Theorem. The solution to the problem

(a) $-z^{\prime}+P^{*} z-\bar{\lambda} z=f$, a.e.,

(b) $z\left(t_{j}+\right)-z\left(t_{j}-\right)=A_{j}^{*} \phi, j=0, \cdots, \infty$, for some parameter $\phi$,

(c) $z\left(0_{-}\right)=0$,

is given by

$$
z(t)=\phi^{*}(t)^{-1} e^{-\bar{\lambda} t} \int_{0}^{t} \phi^{*}(s) e^{\bar{\lambda} s} d F(s) \phi-\phi^{*}(t)^{-1} e^{-\bar{\lambda} t} \int_{0}^{t} \phi^{*}(s) e^{\bar{\lambda} s} f(s) d s .
$$

Proof. Again this easily follows by inspection.

If $\lambda$ is not an eigenvalue of $L$, then the parameter $\phi$ may be determined. The result is the solution represented by using the Green's function $G$.

These equations were studied earlier by Halanay and Moro [5].

3.2. Theorem. The solution $z$ to (a), (b), (c) of Theorem 3.1 is an eigenfunction of $L^{*}$ if and only if

$$
\operatorname{det}\left[U\left(\phi(t) e^{\lambda t}\right)\right]=0,
$$

that is, if and only if $\lambda$ is an eigenvalue of $L$. 
Proof. $z$ is an eigenfunction of $L^{*}$ if and only if $z(1+)=0$, or

$$
\int_{0-}^{1+} \phi^{*}(s) e^{\bar{\lambda} s} d F(s) \phi=\left[\sum_{j=0}^{\infty} \phi^{*}\left(t_{j}\right) e^{\bar{\lambda} t_{j}} A_{j}^{*}\right] \phi=\left[\sum_{j=0}^{\infty} A_{j} \phi\left(t_{j}\right) e^{\lambda t}\right]^{*} \phi=0 \text {. }
$$

This occurs if and only if $\operatorname{det}\left[U\left(\phi(t) e^{\lambda t}\right)\right]=0$. In that case the eigenfunction is of the form given by Theorem 3.1.

Thus for each value $\lambda$ in the point spectrum of $L, \bar{\lambda}$ is in the point spectrum of $L^{*}$. There is no residual or continuous spectrum for $L$ or $L^{*}$. The spectrum for each is purely point spectrum.

The eigenmanifolds for $L$ and $L^{*}$, which occur when $\lambda$ is a multiple root of $\operatorname{det}\left[U\left(\phi(t) e^{\lambda t}\right)\right]=0$, are most easily generated as residues of the Green's function, when considered as a meromorphic function of $\lambda$. Specifically, we wish to evaluate the contour integral

$$
(1 / 2 \pi i) \oint\left[G(\lambda, t, s) /\left(\lambda-\lambda_{0}\right)\right] d \lambda
$$

around an appropriate sequence of contours which approach $\infty$. The resulting residues exhibit the eigenmanifolds and generate an expansion of the Green's function.

3.4. Theorem. Let $\lambda_{0}$ be in the resolvent set of $L$, and let $\lambda_{n}$ be an m-fold zero of $\operatorname{det}\left[U\left(\phi(t) e^{\lambda t}\right)\right]=0$. Near $\lambda_{n}$ let

$$
\begin{aligned}
\phi(t) e^{\lambda t} & =\sum_{j=0}^{\infty} u_{j}\left(\lambda-\lambda_{n}\right)^{j}, \\
M(t, s) \phi(s)^{-1} e^{-\lambda s} & =\sum_{k=0}^{\infty} v_{k}^{*}\left(\lambda-\lambda_{n}\right)^{k}, \\
\left\{U\left(\phi(t) e^{\lambda t}\right)\right\}^{-1} & =\sum_{l=0}^{\infty} U_{l}\left(\lambda-\lambda_{n}\right)^{l-m} .
\end{aligned}
$$

Then the residue of $G(\lambda, t, s) /\left(\lambda-\lambda_{0}\right)$ at $\lambda_{n}$ is

$$
-\sum_{j=0}^{m=1} \sum_{k=0}^{m-1-j} \sum_{l=0}^{m-1-j-k} \frac{u_{j} U_{l} v_{k}^{*}}{\left(\lambda_{0}-\lambda_{n}\right)^{m-j-k-l}} .
$$

Proof. Using the expansions above, we compute

$$
\begin{aligned}
\frac{G(\lambda, t, s)}{\left(\lambda-\lambda_{0}\right)} & =-\sum_{j=0}^{\infty} \sum_{k=0}^{\infty} \sum_{l=0}^{\infty} \sum_{p=0}^{\infty} \frac{u_{j} U_{l} v_{k}^{*}\left(\lambda-\lambda_{n}\right)^{j+k+l+p-m}}{\left(\lambda_{0}-\lambda_{n}\right)^{p+1}} \\
& =-\sum_{q=0}^{\infty} \sum_{j=0}^{q} \sum_{k=0}^{q-j} \sum_{l=0}^{q-j-k} \frac{u_{j} U_{l} v_{k}^{*}\left(\lambda-\lambda_{n}\right)^{q-m}}{\left(\lambda_{0}-\lambda_{n}\right)^{q+1-j-k-l}} .
\end{aligned}
$$


The residue, which occurs when $q=m-1$, is the expression above.

3.5. Theorem. Let $\lambda_{n}$ be an m-fold zero of $\operatorname{det}\left[U\left(\phi(t) e^{\lambda t}\right)\right]$. Then the elements $u_{j}, j=0, \cdots, m-1$, are in $D$. The elements $v_{k}, k=0, \cdots, m-1$, are functions of $s$ alone and are in $D^{*}$.

Proof. These properties are inherited from the Green's function, since at an $m$-fold zero of $\operatorname{det}\left[U\left(\phi(t) e^{\lambda t}\right)\right], \phi(t) e^{\lambda t}, M(t, s) \phi(s)^{-1} e^{-\lambda s}$ and their first $m-$ 1 derivatives are the same for $s>t$ and $s<t$. The only difficulty is in showing that the boundary conditions are satisfied. This also follows from the assumption that $\lambda_{n}$ is an $m$-fold zero.

3.6. Theorem. Let $\lambda_{n}$ be an $m$-fold zero of $\operatorname{det}\left[U\left(\phi(t) e^{\lambda t}\right)\right]$. Then the elements $u_{j}, j=0, \cdots, m-1, v_{k}, k=0, \ldots, m-1$, satisfy

$$
\begin{aligned}
\left(L-\lambda_{n} I\right) u_{0} & =0, & & \\
\left(L-\lambda_{n} I\right) u_{j} & =u_{j-1}, & & j=1, \cdots, m-1, \\
\left(L^{*}-\bar{\lambda}_{n} I\right) v_{0} & =0 & \text { a.e., } & \\
\left(L^{*}-\bar{\lambda}_{n} I\right) v_{k} & =v_{k-1} & \text { a.e., } & k=1, \cdots, m-1 .
\end{aligned}
$$

Proof. For $\lambda$ near $\lambda_{n}, L G=\lambda G$, so $\left(L-\lambda_{n} I\right) G=\left(\lambda-\lambda_{n}\right) G$. Inserting the expansion for $G$, we find

$$
\begin{aligned}
\sum_{j=0}^{\infty} \sum_{l=0}^{\infty} \sum_{k=0}^{\infty}\left(L-\lambda_{n} l\right) u_{j} U_{l} v_{k}^{*}\left(\lambda-\lambda_{n}\right)^{j+l+k-m} \\
=\sum_{j=0}^{\infty} \sum_{l=0}^{\infty} \sum_{k=0}^{\infty} u_{j} U_{l} v_{k}^{*}\left(\lambda-\lambda_{n}\right)^{(j+1)+l+k-m}, \\
=\sum_{j=1}^{\infty} \sum_{l=0}^{\infty} \sum_{k=0}^{\infty} u_{j-1} U_{l} v_{k}^{*}\left(\lambda-\lambda_{n}\right)^{j+l+k-m} .
\end{aligned}
$$

The result then follows from equating coefficients. The proof for the $v$ 's is similar.

3.7. Theorem. Let $\lambda_{n}$ be an m-fold zero of $\operatorname{det}\left[U\left(\phi(t) e^{\lambda t}\right)\right]$. Then $\left\{u_{j}\right\}_{j=0}^{m-1}$ and $\left\{v_{k}\right\}_{k=0}^{m-1}$ form linearly independent sets.

Proof. Suppose for some $J, u_{J}=\Sigma_{j=0}^{J-1} c_{j} u_{j}$. Then applying $\left(L-\lambda_{n} I\right), J$ times, we find $u_{0}=0$. Similarly $u_{1}, \cdots, u_{J}$ are all 0 . This is a contradiction. Again, the proof for the $v$ 's is similar. 
Thus for each eigenvalue $\lambda_{n}$ of $L$, there exists a chain of eigenfunctions $u_{0}, \cdots, u_{m-1}$, with a corresponding chain for $L^{*}, v_{0}, \cdots, v_{m-1}$, having the properties just described. We call the subspaces generated by these chains eigenmanifolds.

3.8. Theorem. The eigenmanifolds for $L$ and $L^{*}$ corresponding to different eigenvalues are mutually orthogonal. The eigenmanifolds, therefore, form a set of biorthogonal manifolds.

Proof. Let us assume that

$$
\left(L-\lambda_{i} l\right) u_{i j}=u_{i j-1}, \quad\left(L^{*}-\bar{\lambda}_{k} I\right) v_{k l}=v_{k l-1},
$$

where $\lambda_{i} \neq \lambda_{k}$. Then

$$
\begin{aligned}
\left(\lambda_{i}-\lambda_{k}\right)\left(u_{i j}, v_{k l}\right) & =\left(\lambda_{i} u_{i j}, v_{k l}\right)-\left(u_{i j}, \bar{\lambda}_{k} v_{k l}\right) \\
& =\left(L u_{i j}, v_{k l}\right)-\left(u_{i j-1}, v_{k l}\right)-\left(u_{i j}, L^{*} v_{k l}\right)+\left(u_{i j}, v_{k l-1}\right) \\
& =-\left(u_{i j-1}, v_{k l}\right)+\left(u_{i j}, v_{k l-1}\right) .
\end{aligned}
$$

An induction on $j$ and $l$ shows these last two terms are also zero. Since $\lambda_{i} \neq \lambda_{k}$, $\left(u_{i j}, v_{k l}\right)=0$.

4. Expansion of the Green's function. We use the standard technique in order to expand the Green's function: contour integration about a sequence of contours which approach $\infty$. In order to see that this process works, we need some preliminary results. We recall that the coefficients $A_{0}$ and $A_{1}$ are nonsingular matrices.

4.1. Lemma. If $|\operatorname{Re}(\lambda)|$ is sufficiently large, then $\lambda$ is in the resolvent set of $L$.

Proof. Let $\operatorname{Re}(\lambda)>0$. Then

$$
\sum_{j=0}^{\infty} A_{j} \phi\left(t_{j}\right) e^{\lambda t_{j}}=e^{\lambda}\left[A_{0} \phi(0) e^{-\lambda}+A_{1} \phi(1)+\sum_{j=2}^{\infty} A_{j} \phi\left(t_{j}\right) e^{\lambda\left(t_{j}-1\right)}\right] \text {. }
$$

As $\operatorname{Re}(\lambda)$ approaches $+\infty$, it is easy to show that $A_{1} \phi(1) e^{\lambda}$, which is nonsingular, is the dominant term.

If $\operatorname{Re}(\lambda)<0$, a similar argument shows that $A_{0} \phi(0)$ dominates as $\operatorname{Re}(\lambda)$ approaches $-\infty$. This, too, is nonsingular.

4.2. Lemma. As $\operatorname{Re}(\lambda)$ approaches $+\infty, G(\lambda, t, s)$ approaches 0 uniformly in $t$ and $s$. 
Proof. If $s \leqq t$, then

$$
\begin{aligned}
G(\lambda, t, s) & =e^{\lambda t} \phi(t)[I+o(1)]^{-1} e^{-\lambda} \phi(1)^{-1} A_{1}^{-1} \sum_{t_{k} \leq s} A_{k} \phi\left(t_{k}\right) e^{\lambda t_{k}} \phi(s)^{-1} e^{-\lambda s} \\
& =e^{\lambda(t-1)} \phi(t)[I+o(1)]^{-1} \phi(1)^{-1} A_{1}^{-1} \sum_{t_{k} \leq s} A_{k} \phi\left(t_{k}\right) \phi(s)^{-1} e^{\lambda\left(t_{k}-s\right)},
\end{aligned}
$$

which approaches 0 as $\operatorname{Re}(\lambda)$ approaches $+\infty$.

If $s>t$,

$$
\begin{aligned}
G(\lambda, t, s) & =-e^{\lambda t} \phi(t)[I+o(1)]^{-1} e^{-\lambda} \phi(1)^{-1} A_{1}^{-1} \sum_{t_{k}>s} A_{k} \phi\left(t_{k}\right) e^{\lambda t_{k}} \phi(s)^{-1} e^{-\lambda s} \\
& =-e^{\lambda(t-s)} \phi(t)[I+o(1)]^{-1} \phi(1)^{-1} A_{1}^{-1} \sum_{t_{k}>s} A_{k} \phi\left(t_{k}\right) \phi(s)^{-1} e^{\lambda\left(t_{k}-1\right)},
\end{aligned}
$$

which also approaches 0 as $\operatorname{Re}(\lambda)$ approaches $+\infty$.

4.3. Lemma. As $\operatorname{Re}(\lambda)$ approaches $-\infty, G(\lambda, t, s)$ approaches 0 uniformly in $t$ and $s$.

Proof. If $s \leqq t$,

$$
\begin{aligned}
G(\lambda, t, s) & =e^{\lambda t} \phi(t)[I+o(1)]^{-1} \phi(0)^{-1} A_{0}^{-1} \sum_{t_{k} \leq s} A_{k} \phi\left(t_{k}\right) e^{\lambda t_{k}} \phi(s)^{-1} e^{-\lambda s} \\
& =e^{\lambda(t-s)}[1+o(1)]^{-1} \phi(0)^{-1} A_{0}^{-1} \sum_{t_{k} \leq s} A_{k} \phi\left(t_{k}\right) \phi(s)^{-1} e^{\lambda t_{k}},
\end{aligned}
$$

which approaches 0 as $\operatorname{Re}(\lambda)$ approaches $-\infty$.

If $s>t$,

$$
\begin{aligned}
G(\lambda, t, s) & =-e^{\lambda t} \phi(t)[I+o(1)]^{-1} \phi(0)^{-1} A_{0}^{-1} \sum_{t_{k}>s} A_{k} \phi\left(t_{k}\right) e^{\lambda t_{k}} \phi(s)^{-1} e^{-\lambda s} \\
& =-e^{\lambda t} \phi(t)[I+o(1)]^{-1} \phi(0)^{-1} A_{0}^{-1} \sum_{t_{k}>s} A_{k} \phi\left(t_{k}\right) \phi(s)^{-1} e^{\lambda\left(t_{k}-s\right)},
\end{aligned}
$$

which approaches 0 as $\operatorname{Re}(\lambda)$ approaches $-\infty$.

We are now ready to perform the contour integration.

4.4. Theorem. Let the eigenvalues of $L$ be denoted by $\left\{\lambda_{n}\right\}_{n=1}^{\infty}$, where their orders $m$, and the elements $u_{j}, U_{l}, v_{k}$ are dependent upon $n$. Then, if $\lambda_{0}$ is in the resolvent set for $L$, 


$$
G\left(\lambda_{0}, t, s\right)=\sum_{n=1}^{\infty}\left[\sum_{j=0}^{m-1} \sum_{k=0}^{m-1-j} \sum_{l=0}^{m-1-j-k} u_{j}(t) U_{l} v_{k}^{*}(s) /\left(\lambda_{0}-\lambda_{n}\right)^{m-j-k-l}\right]
$$

uniformly in $t$ and $s$, a.e.

Proof. We consider $(1 / 2 \pi i) \int_{C}\left[G(\lambda, t, s) /\left(\lambda-\lambda_{0}\right)\right] d \lambda$ over the contour consisting of the following: along a semicircle centered at $(r, 0)$ with radius $R$ from $(r,-R)$ to $(r, R)$ in a positive direction, horizontally from $(r, R)$ to $(-r, R)$, along a semicircle centered at $(-r, 0)$ with radius $R$ from $(-r, R)$ to $(-r,-R)$ in a positive direction, finally horizontally from $(-r,-R)$ to $(r,-R)$. If $r$ is chosen sufficiently large, the integrals on the semicircles can be made arbitrarily small by using Lemmas 4.2 and 4.3 .

We now wish to show that when bounded away from the eigenvalues $G$ is uniformly bounded. In this event the integrals over the horizontal portions will be bounded by a constant times $\Delta \theta$, the change in $\arg \lambda$, over these portions. Since this approaches 0 as $R$ approaches $\infty$, these integrals will also become arbitrarily small.

The only portion of the Green's function to give any difficulty is the inverse of

$$
f(\lambda)=\operatorname{det}\left[U\left(\phi(t) e^{\lambda t}\right)\right]=\operatorname{det}\left[\sum_{j=0}^{\infty} A_{j} \phi\left(t_{j}\right) e^{\lambda t_{j}}\right] .
$$

It is well known that its zeros are isolated, have no finite accumulation point, and are uniformly spaced throughout a vertical strip. If the zeros are isolated by small circles of radius $\delta$, then according to Levin, Zeros of entire functions, $\mathrm{p}$. 268 , there exists a constant $m(\delta)>0$ such that $|f(\lambda)| \geq m(\delta)$ outside those circles. Thus $f(\lambda)^{-1}$ is bounded.

The final result now follows from the Cauchy residue theorem.

5. Eigenfunction expansions. There exist two eigenfunction expansions: one for elements in $D$, one for elements in $D^{*}$. They are derived in the standard way. In addition Parseval's equality can be stated for pairs of elements, one in $D$, the other in $D^{*}$.

5.1. Theorem. Let $y$ be in D. Then

$$
y(t)=-\sum_{n=1}^{\infty}\left[\sum_{j=0}^{m-1} \sum_{k=0}^{m-1-j} u_{j}(t) U_{m-1-j-k} \int_{0}^{1} v_{k}^{*}(s) y(s) d s\right],
$$

where the equality is uniform in $t$. 
Proof. Let $\left(L-\lambda_{0} I\right) y=f$ where $\lambda_{0}$ is in the resolvent set for $L$. Then

$$
\begin{aligned}
y(t) & =\int_{0}^{1} G(\lambda, t, s) f(s) d s \\
& =\sum_{n=1}^{\infty}\left[\sum_{j=0}^{m-1} \sum_{k=0}^{m-1-j} \sum_{l=0}^{m-1-j-k} u_{j}(t) U_{l} \int_{0}^{1} v_{k}^{*}(s) f(s) d s /\left(\lambda_{0}-\lambda_{n}\right)^{m-j-k-l}\right] .
\end{aligned}
$$

Now

$$
\begin{aligned}
\int_{0}^{1} v_{k}^{*}(s) f(s) d s & =\left(f, v_{k}\right)=\left(\left[L-\lambda_{0} I\right] y, v_{k}\right)=\left(y,\left[L^{*}-\bar{\lambda}_{0} I\right] v_{k}\right) \\
& =\left(y,\left[L^{*}-\bar{\lambda}_{n}\right] v_{k}\right)+\left(y,\left[\bar{\lambda}_{n}-\bar{\lambda}_{0}\right] v_{k}\right)=\left(y, v_{k-1}\right)+\left[\lambda_{n}-\lambda_{0}\right]\left(y, v_{k}\right) .
\end{aligned}
$$

Inserting this in the expression for $y$,

$$
y(t)=\sum_{n=1}^{\infty}\left[\sum_{j=0}^{m-1} \sum_{k=0}^{m-1-j} \sum_{l=0}^{m-1-j-k} u_{j}(t) U_{l}\left\{\frac{\left(y, v_{k-1}\right)}{\left(\lambda_{0}-\lambda_{n}\right)^{m-j-k-l}}-\frac{\left(y, v_{k}\right)}{\left(\lambda_{0}-\lambda_{n}\right)^{m-1-j-k-l}}\right\}\right] .
$$

The left side is independent of $\lambda_{0}$. Thus, those terms on the right which involve $\lambda_{0}$ must equal zero, when added. $y(t)$ is equal to only those which are independent of $\lambda_{0}$. Hence

$$
y(t)=-\sum_{n=1}^{\infty}\left[\sum_{j=0}^{m-1} \sum_{k=0}^{m-1-j} u_{j}(t) U_{m-1-j-k} \int_{0}^{1} v_{k}^{*}(s) y(s) d s\right] .
$$

Since both sides are continuous in $t$, in addition to being equal in $H$, they are equal uniformly.

5.2. Theorem. Let $z$ be in $D^{*}$. Then

$$
z(s)=-\sum_{n=1}^{\infty}\left[\sum_{j=0}^{m-1} \sum_{k=0}^{m-1-j} v_{k}(s) U_{m-1-j-k}^{*} \int_{0}^{1} u_{j}^{*}(t) z(t) d t\right]
$$

where the equality is a.e. in s.

The proof is similar to that of the preceding theorem.

5.3. Theorem (Parseval's Equality). Let $y$ be in $D$ and $z$ be in $D^{*}$. Then

$$
(y, z)=-\sum_{n=1}^{\infty}\left[\sum_{j=0}^{m-1} \sum_{k=0}^{m-1-j} \int_{0}^{1} z^{*}(t) u_{j}(t) d t U_{m-1-j-k} \int_{0}^{1} v_{k}^{*}(s) y(s) d s\right] .
$$

This easily follows from either of the preceding two theorems.

6. Remarks. The conditions that $A_{0}$ and $A_{1}$ be nonsingular are essential. Without them the eigenvalues are not restricted to a vertical strip in the complex 
plane. The requirement that $\sum_{j=0}^{\infty}\left\|A_{j}\right\|<\infty$ is also crucial. If this series diverges, the whole nature of the problem changes. For example, eigenvalues and eigenfunctions fail to exist.

What actually occurs when $\sum_{j=1}^{\infty}\left\|A_{j}\right\|$ diverges is not yet known. In addition what sort of generalization of this problem is classically selfadjoint is not known. What occurs when the boundary condition has the form

$$
\sum_{j=0}^{\infty} A_{j} y\left(t_{j}\right)+\int_{0}^{1} K(t) y(t) d t=0
$$

can be surmised, but has not yet been proved. Finally, what sort of generalization with integral plus series boundary condition is classically selfadjoint can only be guessed at present. For further information we suggest [2], [6] and [8].

\section{REFERENCES}

1. G. D. Birkhoff and R. E. Langer, The boundary problems and developments associated with a system of ordinary differential equations of the first order, Proc. Amer. Acad. Arts Sci. 58 (1923), 51-128.

2. R. N. Bryan, A nonhomogeneous linear differential system with interface conditions, Proc. Amer. Math. Soc. 22 (1969), 270-276. MR 39 \#3078.

3. R. H. Cole, The expansion problem with boundary conditions at a finite set of points, Canad. J. Math. 13 (1961), 462-479. MR 23 \#A1090.

4. G. B. Green and A. M. Krall, Linear differential systems with infinitely many boundary points, Ann. Mat. Pura Appl. (to appear).

5. A. Halanay and A. Moro, $A$ boundary value problem and its adjoint, Ann. Mat. Pura Appl. (4) 79 (1968), 399-411. MR 38 \#2371.

6. A. M. Krall, Differential-boundary operators, Trans. Amer. Math. Soc. 154 (1971), 429-458. MR $42 \# 6328$.

7. - Self-adjoint boundary value problems with infinitely many boundary points, Ann. Mat. Pura Appl. (to appear).

8. D. H. Tucker, Boundary value problems for linear differential systems, SIAM J. Appl. Math. 17 (1969), 769-783. MR 40 \#5958.

DEPARTMENT OF MATHEMATICS, PENNSYLVANIA STATE UNIVERSITY, UNIVERSITY PARK, PENNSYLVANIA 16802 